\title{
Aphasia as a Temporal Information Processing Disorder
}

\author{
Elzbieta Szelag ${ }^{* \dagger}$, Aneta Szymaszek ${ }^{*, \dagger}$, and Anna Oron*
}

In the Millennium issue of the Brain and Language journal, several articles emphasised the importance of studying temporal aspects of information processing and their close associations with language processing. One article even stated that “...on space, time and language: for the next century, timing is (almost) everything" (Osterhout, 2000).

Temporal information processing (TIP) mechanisms have been identified as the neural basis for many higher cognitive functions, such as language, memory, new learning, attention, emotional evaluation, motor control, executive system, planning of activities or decision making. All these functions can be characterized by specific temporal dynamics at different ranges of TIP. Such temporal constraints can be observed both within millisecond and multisecond time ranges, which create pre-semantically defined (i.e., prior to any semantic evaluation) temporal 'windows' for cognitive processing (Pöppel, 1997, 2004, 2009). Such temporal dynamics appear to be a general property of human cognition. It provides a structure not only for language, but also for any perceptual or motor act, as well as any functional state characterized apparently by spatiotemporal patterns of behaviour, which are evidenced in neuroimaging studies.

Strong experimental evidence supporting this conceptual notion comes from the language domain in which temporal structure has been well documented by every day observations of fluent speech, as well as linguistic and psychophysical data. Using different techniques and populations, experimental studies have consistently indicated that the left hemispheric superiority for processing verbal information may reflect more specific control of temporal cues for which human speech is one example (Szelag et al., 2008, 2011; Wittmann et al., 2004). This evidence can be supported by clinical data, indicating that certain left hemispheric lesions selectively affect temporal mechanisms (see Table 12.2).

\footnotetext{
* Laboratory of Neuropsychology, Nencki Institute of Experimental Biology, Warsaw, Poland.

$\dagger$ University of Social Sciences and Humanities, Warsaw, Poland.

(C) ELZBiEtA SZElAG, ANETA SZYMASZEK, \& ANNA ORON, 2015 | DOI 10.1163/9789004230699_013 This is an open access chapter distributed under the terms of the Creative Commons 
In the present chapter, we concentrate on language functions, especially on TIP and acquired language disorders following injuries to the brain, resulting in aphasia. As many different methodologies have been employed in studies on TIP, we will also discuss theoretical perspectives in relation to these findings. The purpose of this chapter is to summarize the results of studies described in the existing literature focusing on TIP in aphasia and to illustrate these data with the results of our own studies.

Language is a highly specialized mental function that is a key factor in social interaction and the evolution of human culture. The broader definition of language in Cognitive Neuroscience is "... a symbolic system used to communicate concrete or abstract meanings, irrespective of the sensory modality employed or the particular means of expression..." (Purves et al., 2008, p. 511). On the other hand, human speech can be defined as vocalized concrete acts of language used in order to communicate (e.g., Price, 2004).

It has been commonly accepted that human language is a complex system, thus, it is not monolithic. Several language functions or sub-systems are incorporated within the framework of network models of language. Accordingly, language includes spoken (expression) or heard (reception) modalities, communication in writing or reading (visual forms), as well as sign language. Although verbal output differs significantly among the ca. 6000 languages existing worldwide, every given natural language contains specific phonology, vocabulary, grammar, and syntactic rules.

For neurolinguists, the classical dichotomy assumes differentiation between expression (production) and reception (understanding). Language expression and our spoken utterances incorporate several processes and sub-functions involved in the production of the verbal output, such as: conceptual preparation including planning a sequence of the speech acts, articulation, verbal fluency, lexicon (lexical encoding), syntax (grammatical encoding), phonology (phonological encoding), prosody, suprasegmental encoding, pragmatics, self-monitoring and repair (Blanken et al., 1993). On the other hand, language reception comprises several processes and mechanisms that allow the listener to understand the speaker's communicative intentions, as well as allow the speaker to understand his or her own speech. The linguistic processing comprises acoustic-phonetic analysis of the speech signal, phonology (phonological decoding), vocabulary (lexical selection), sentences, syntax (grammatical decoding), and, finally, interpretation of discourse. 
It should be stressed that these processes do not work sequentially one after another. On the contrary, parallel processing occurs in both speaking and listening. Direct feedback interactions between particular components of speech production or speech understanding processes have been heavily debated. There is only some evidence on the existence of highly limited feedback from phonological to grammatical encoding in speech production (Blanken et al., 1993).

Despite diversity of speech sounds, grammar, and syntax among different mother tongues, some important generalization regarding the neuroanatomical representation and neural mechanisms underlying language communication are possible and, thus, reviewed below.

Epidemiology, Definitions, and Clinical Syndromes of Aphasia

The term aphasia denotes acquired disturbances of language processing, resulting from brain damage. Aphasia is a complex language disorder and refers to deficits apparent in speech, writing or reading, caused by an injury to brain regions specialized for these functions. This definition contains four crucial aspects, namely: (1) aphasia is acquired; (2) aphasia is a consequence of brain damage; (3) aphasia affects language processes; (4) other cognitive functions are relatively spared.

Several types of aphasia classification and classes of aphasic syndromes have been proposed, among other by Luria, Geschwind, Goodglass, Kertesz, and other researchers. The following are the main aphasic syndromes: global aphasia, Broca's aphasia, Wernicke's aphasia, amnestic aphasia, conduction aphasia, and transcortical aphasias. The classic location of brain damage in these aphasia types as well as the main language deficits are summarized in Table 12.1.

Nowadays, aphasia results predominantly from brain stroke and approximately $60 \%$ of stroke patients suffer from aphasia. The most frequent cause of aphasia is ischemic infarction in the territory of supply of the left middle cerebral artery. Other causes are haemorrhages, tumour, trauma, cerebral infection or atrophic pathology.

It is estimated that up to 270.000 patients with stroke-related aphasia are diagnosed worldwide each year (Szaflarski et al., 2011). Only a minority of these patients recover completely from their language deficits. Examinations of typical recovery curves reported in the literature have revealed that only $25 \%$ of patients have a chance for full restoration of disturbed language functions. These data indicate that aphasia still constitutes a major medical and social 


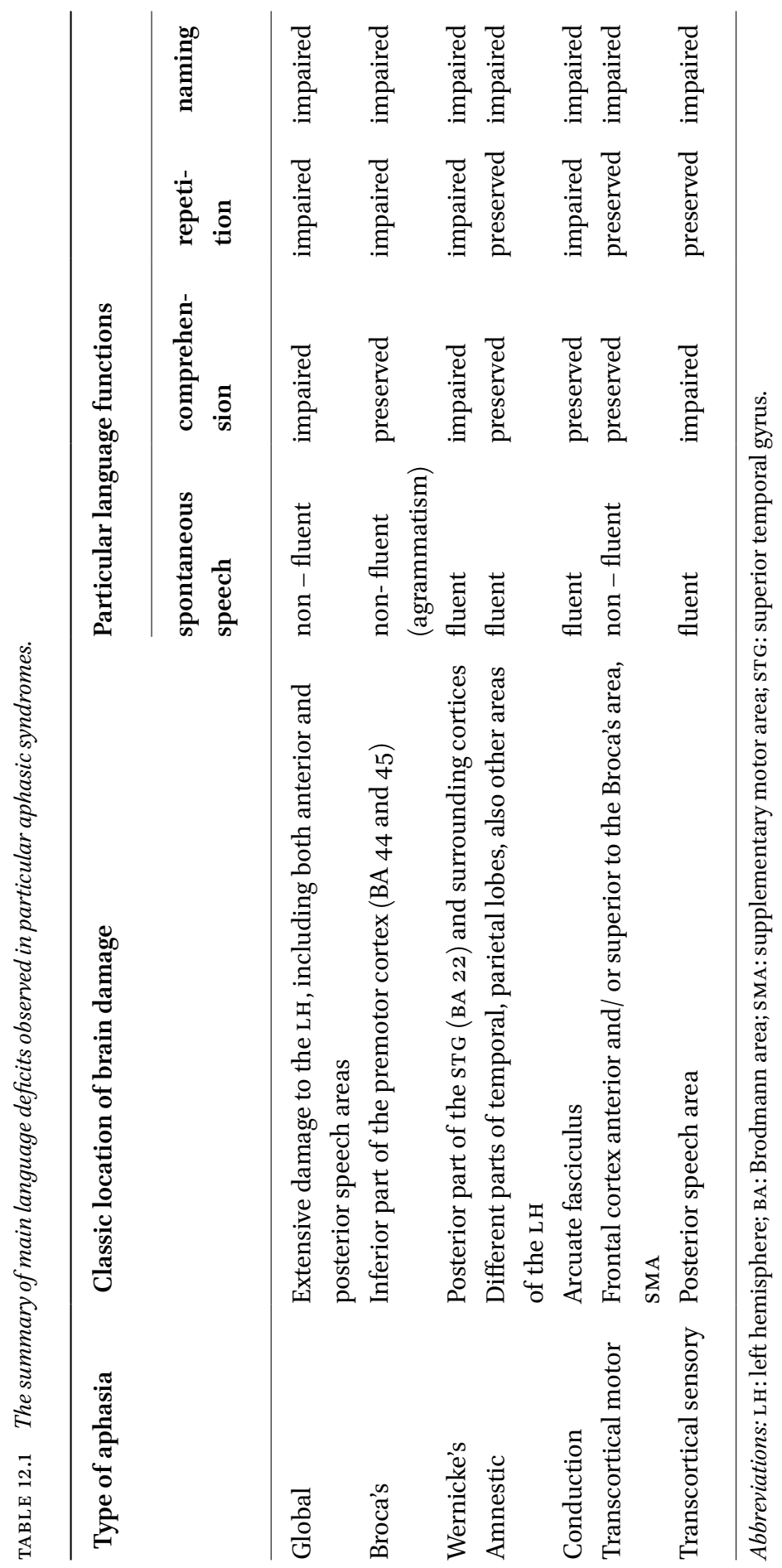


problem in modern society. This should inspire researchers worldwide to elaborate new techniques of language rehabilitation.

Current knowledge on understanding cerebral representation of language comes from 4 main groups of evidence: (1) clinical observations of language deficits following acquired lesions to the brain; (2) electrophysiological mapping of brain areas as an adjunct to various neurosurgical procedures; (3) 'splitbrain' studies of patients whose cerebral hemispheres were surgically disconnected as a treatment of epilepsy; (4) experimental studies employing many different laboratory techniques (e.g., psychophysical, behavioural, molecular, biochemical, and neuroimaging).

\subsection{Clinical Observations of Language Deficits}

An important key generalization is that in the vast majority of individuals the left brain hemisphere is a primary locus of language communication. Accumulating evidence has indicated such cerebral representation in $96 \%$ of right-handed and in ca. $70 \%$ of left-handed individuals (e.g., Springer and Deutsch, 1998). Another generalization is that brain areas involved in language are closely related to the sensory (auditory and visual) and motor regions that process non-speech signals, as well as generate the non-speech motor acts (Springer and Deutsch, 1998).

\subsection{Electrophysiological Mapping of Brain Areas}

Further insight into more detailed mapping of language functions within the left hemispheric cortical areas comes from electrical stimulation of cerebral cortex during surgery by Penfield and Roberts (1959). The reason for such surgery is usually brain tumour or searching for epileptic focus before removing diseased brain tissue. Such stimulations were performed in awake and conscious patients and allowed the surgeon to localize cortical areas that interfere with speech during stimulation. These observations allowed exact location of language and indicated that several left hemispheric regions control different language functions. These large regions are usually outside the 'classic' language area described by Paul Broca and Carl Wernicke in the late 1800 (Blanken et al., 1993). Despite individual differences in the borders of the region supporting language, it comprises usually parts of the left hemispheric frontal, temporal, and parietal lobes, specifically, large regions of perisylvian cortices for both language production and comprehension. 


\section{3 'Split-brain' Studies}

Commissurotomy refers to complete surgical sections of the corpus callosum, anterior commissure, dorsal, and ventral hippocampal commissures and, in some cases, the massa intermedia. It resulted in total isolation of cortical areas of two hemispheres. Following complete cerebral commissurotomy, the clear hemispheric specialization was observed as the 'split-brain' phenomenon provided clear evidence on independent contribution of each hemisphere to language processing as well as special behaviour and capacity of isolated hemispheres.

In summary, the left hemisphere represented language competence observed in speaking, hearing, reading or writing, but some limited right hemispheric capacities in language comprehension and production were also evidenced (Zaidel, 2001; Zaidel et al., 2003).

\subsection{Experimental Studies}

The results of detailed mapping of language functions derived from brain stimulation during surgery were further supported by non-invasive brain imaging techniques, using electrophysiological methods, positron emission tomography (PET), functional magnetic resonance imaging (fMRI), magnetoencephalography (MEG) or suppression of language functions during transcranial magnetic stimulation (TMS). All these modern techniques showed activity of various areas in different language tasks, indicating a broader picture of the way language is processed.

\subsection{Summary of Cortical Regions Supporting Language}

A detailed characteristic of cerebral representation of language is beyond the scope of the present chapter but has been described for example by Scott (2012) and Turken and Donkers (2011). We summarized briefly the main role of cortical regions supporting language communication, focussing only on clinical-pathological data of patients with lesions of the specific brain areas. Next, we referred these clinical data to the neural basis for language production and comprehension. It is evident that the clinical data were further confirmed by results of modern neuroimaging studies. Such approach is justified by the topic of the present chapter in which we concentrate on the dissociation between different ranges of TIP or 'temporal windows' (i.e., processing of information within millisecond $v s$. multisecond time domains) in case of expressive and receptive deficits observed in particular aphasia types. In this approach, therefore, we concentrate on the left frontal lobe where the motor functions are carried out controlling language expression, as well as on the left posterior regions of the temporal lobe in which the auditory functions are represented, controlling language reception. Moreover, in language 
communication subcortical structures are involved, e.g., basal ganglia, insula, cingulate gyrus or cerebellum, as well as other structures supporting writing, reading, verbal memory, word finding, repetition, verbal fluency, programming of longer verbal utterances, affective control, and articulation. The right hemispheric contribution seems also important.

The neural basis for producing language comprises Broca's area, i.e., inferior part of the premotor cortex (inferior frontal gyrus) of the left frontal lobe. This region includes Brodmann areas 44 and 45 . Initial evidence on the involvement of this region in producing language was provided by the French anatomist and neurologist - Paul Broca in 1861. He described a patient who suffered from acquired brain damage to this region, resulting in disability to produce fluent speech. The lesion's location was confirmed by Broca in post-mortem brain examination.

The importance of this region is vividly evidenced in patients suffering from motor aphasia (other names: efferent motor aphasia, Broca's aphasia, production aphasia, or nonfluent aphasia), a common neurologic disorder, resulting in inability to organize and control the linguistic content of the utterance with relatively preserved auditory comprehension. The typical patient suffering from a lesion in the Broca's area cannot express thoughts appropriately, because of the loss of the meaningful fluent verbal output, reduced phrase length (so called 'telegraphic style' of the verbal output) and agrammatism, i.e., disrupted rules of grammar and syntax, even though the verbal apparatus and articulatory movements remained intact.

The core symptom in Broca's aphasia is a disruption of brain mechanisms responsible for producing phonemes, as well as combining phonemes into morphemes and words into sentences. Thus, in Broca's aphasia the efferent pathway from the Broca's area to the peripheral articulatory apparatus is disrupted. Generally, such patients produce structurally incorrect utterances, nonsense syllables, transpose words with some sensible meaning. Thus, a listener may have an impression that the patients knew what they intended to say.

Broca's aphasia encompasses interactions of several impairments, including defects in linguistic formulation, motor programming, initiation and maintenance of verbal output, and affective control. Thus, selective damage to the posterior inferior frontal cortex does not produce the full motor syndrome, but rather Broca's aphasia is caused by extensive damage to several neighbouring frontal regions.

On the other hand, an important contribution indicating the neuroanatomical basis of language comprehension was made first by the German psychiatrist - Carl Wernicke in 1874. He described for the first time a patient who produced fluent utterances with appropriate grammar and syntax, but could not understand heard (or written) communications addressed to him by other 
speakers. These deficits were caused by damage to the left hemispheric temporal lobe, more specifically, to the posterior part of the superior temporal gyrus, which is referred to, in honour of its discoverer, as Wernicke's area. According to the Brodmann map it comprises area 22, but often also surrounding temporal and parietal cortices. The lesions in this part of the brain caused Wernicke's aphasia (other names: receptive aphasia, sensory aphasia or fluent aphasia).

In contrast to Broca's aphasia, patients suffering from lesions affecting Wernicke's area cannot understand the speech of other people, as well as their own speech. As a consequence, utterances generated by such patients have little (or even not at all) sense because phonemes, syllables, and words are not correctly linked. In more severe forms of this disorder, the verbal output is named 'jargon aphasia', as the listener cannot understand it at all. Wernicke's aphasia can be characterized by fluent speech, adequate syntax and grammar, but inappropriate choice of words (paraphasia) and little spontaneous repetitions. A core symptom of Wernicke's aphasia is disruption of phonemic hearing, i.e., the ability of analysis and synthesis of speech sounds (phonological decoding). For example, a patient with such disruption cannot properly decode phonemes (consonants and vowels) and, as a consequence, is not able to properly decode words and sentences. The impaired phonological hearing results, therefore, in disordered comprehension. The schematic description of classic aphasic syndromes is given in Figure 12.1 (compare also Table 12.2 and Section 7, below).

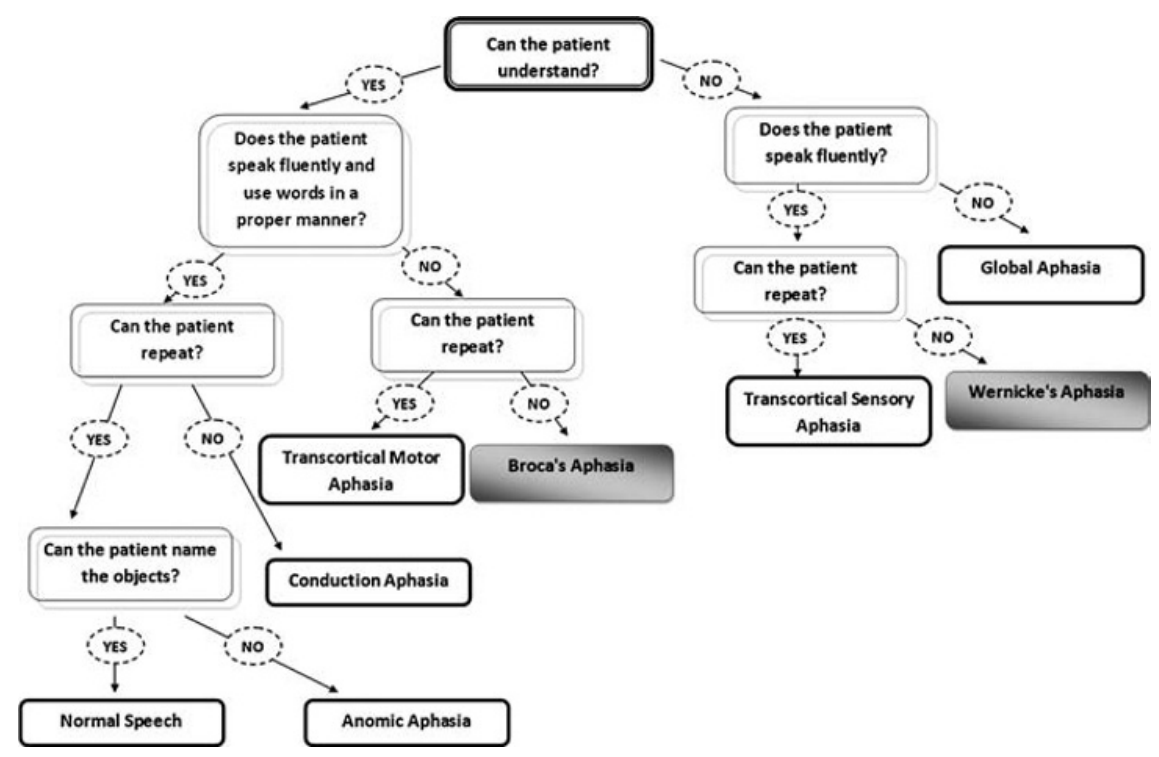

FIGURE 12.1 The differential diagnosis of main aphasic syndromes. 


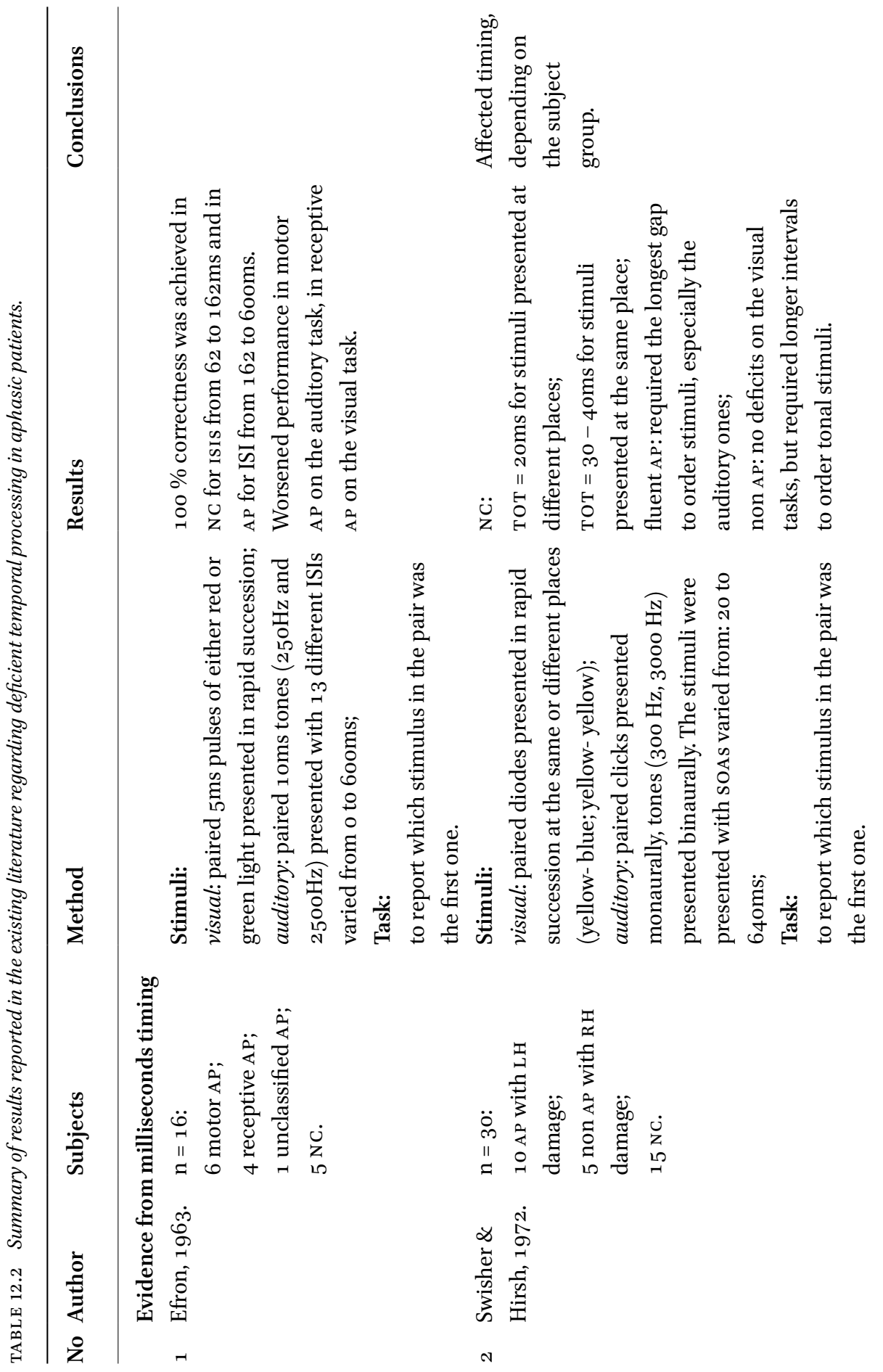



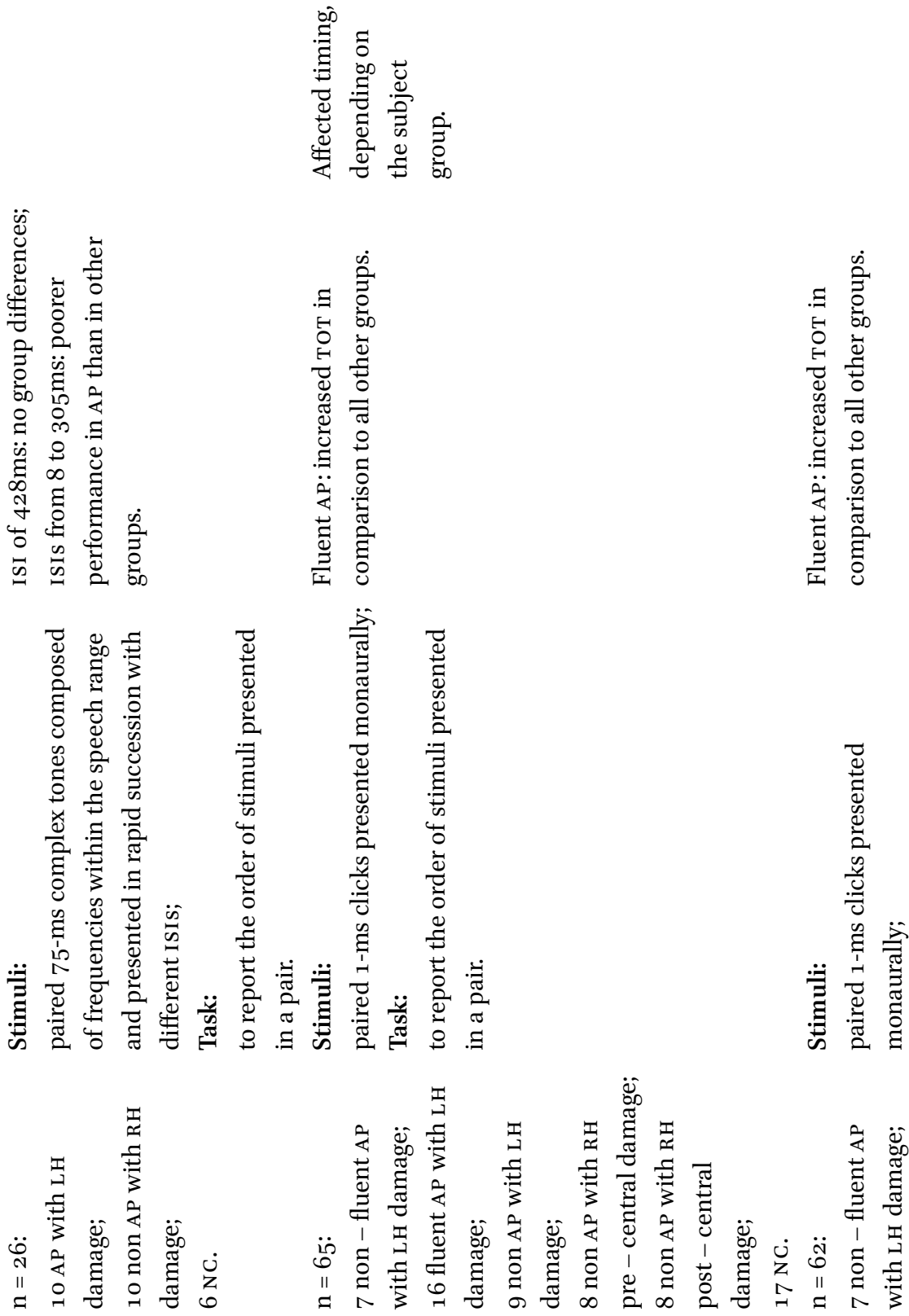

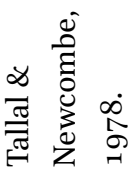

$m$

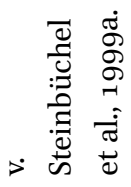

४

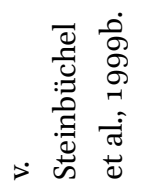

ת 

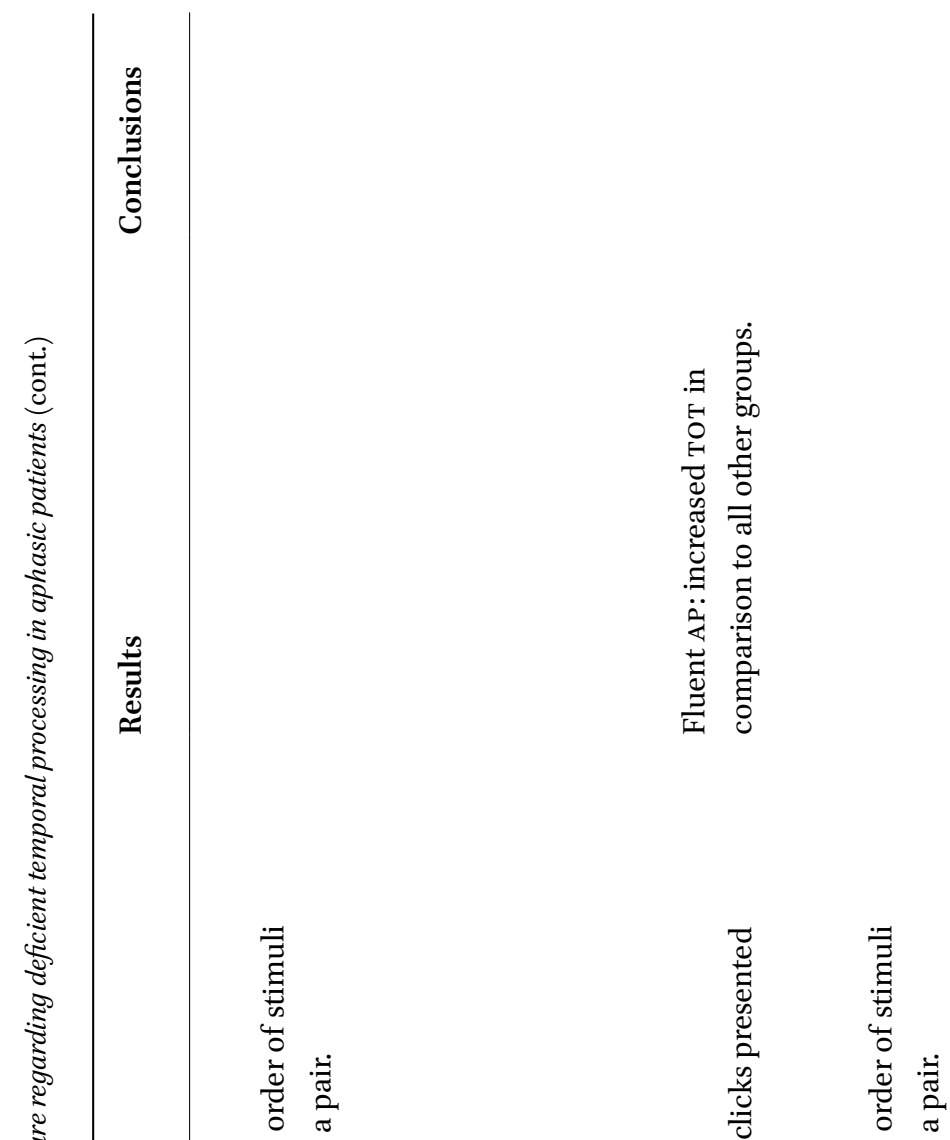

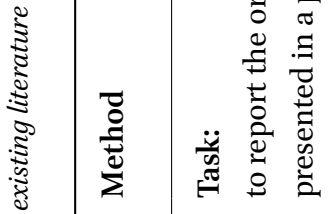

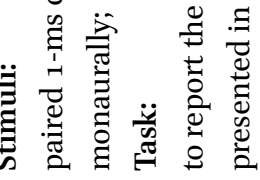

ฐ

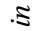

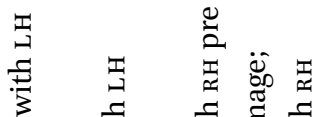

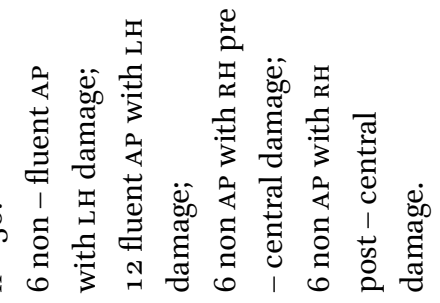

苞

6 


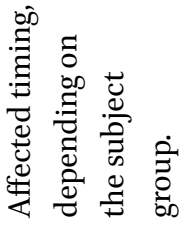

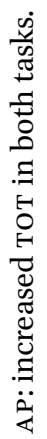

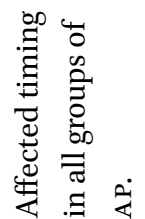

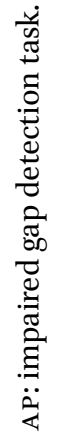
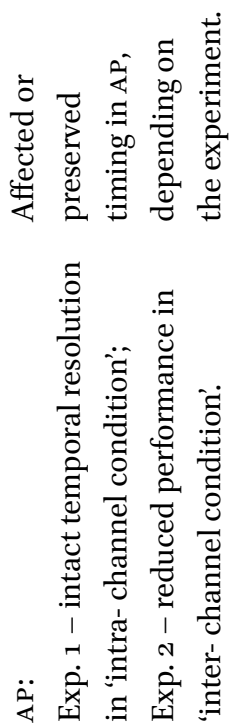
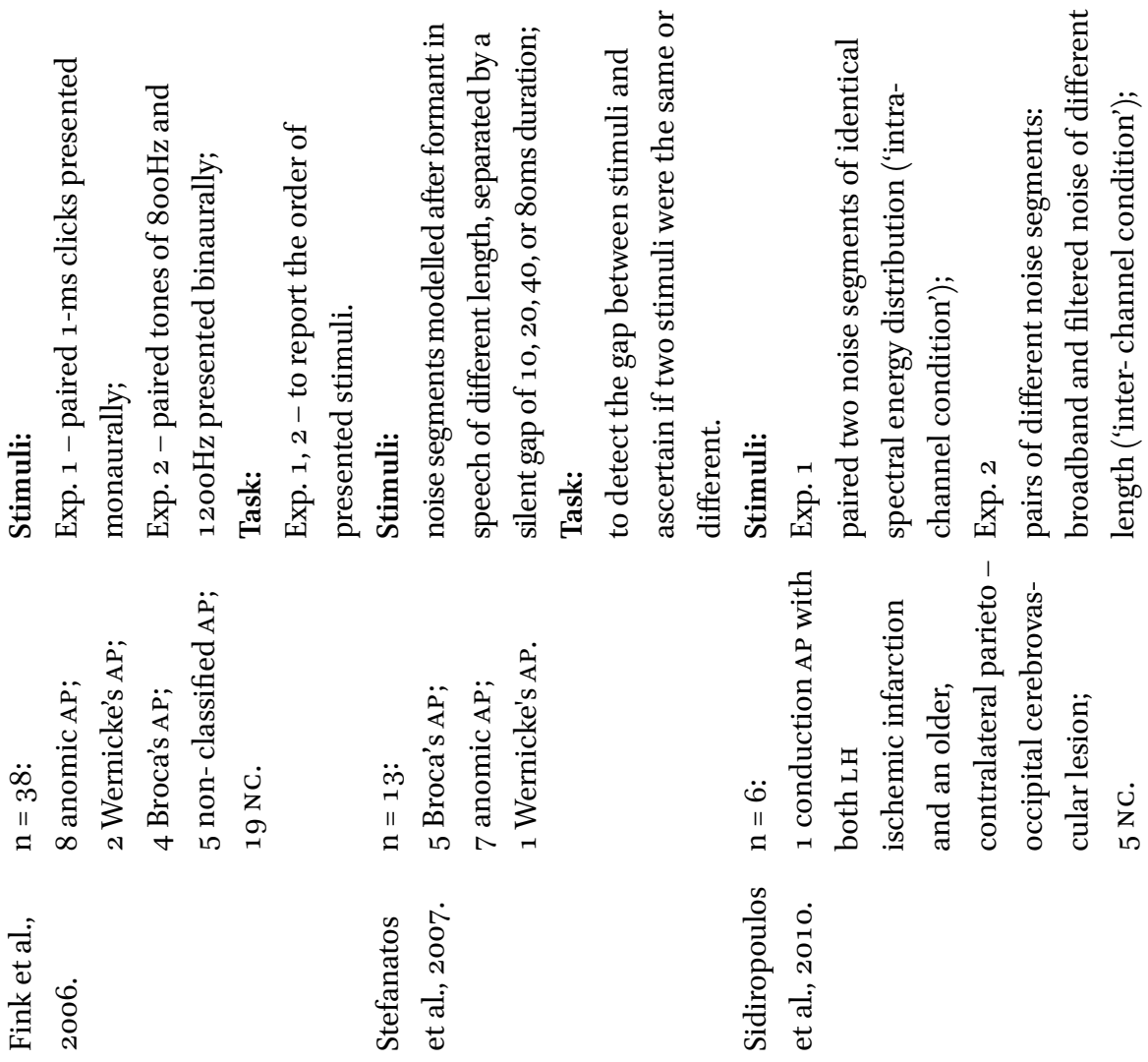

$N$

$\infty$ 


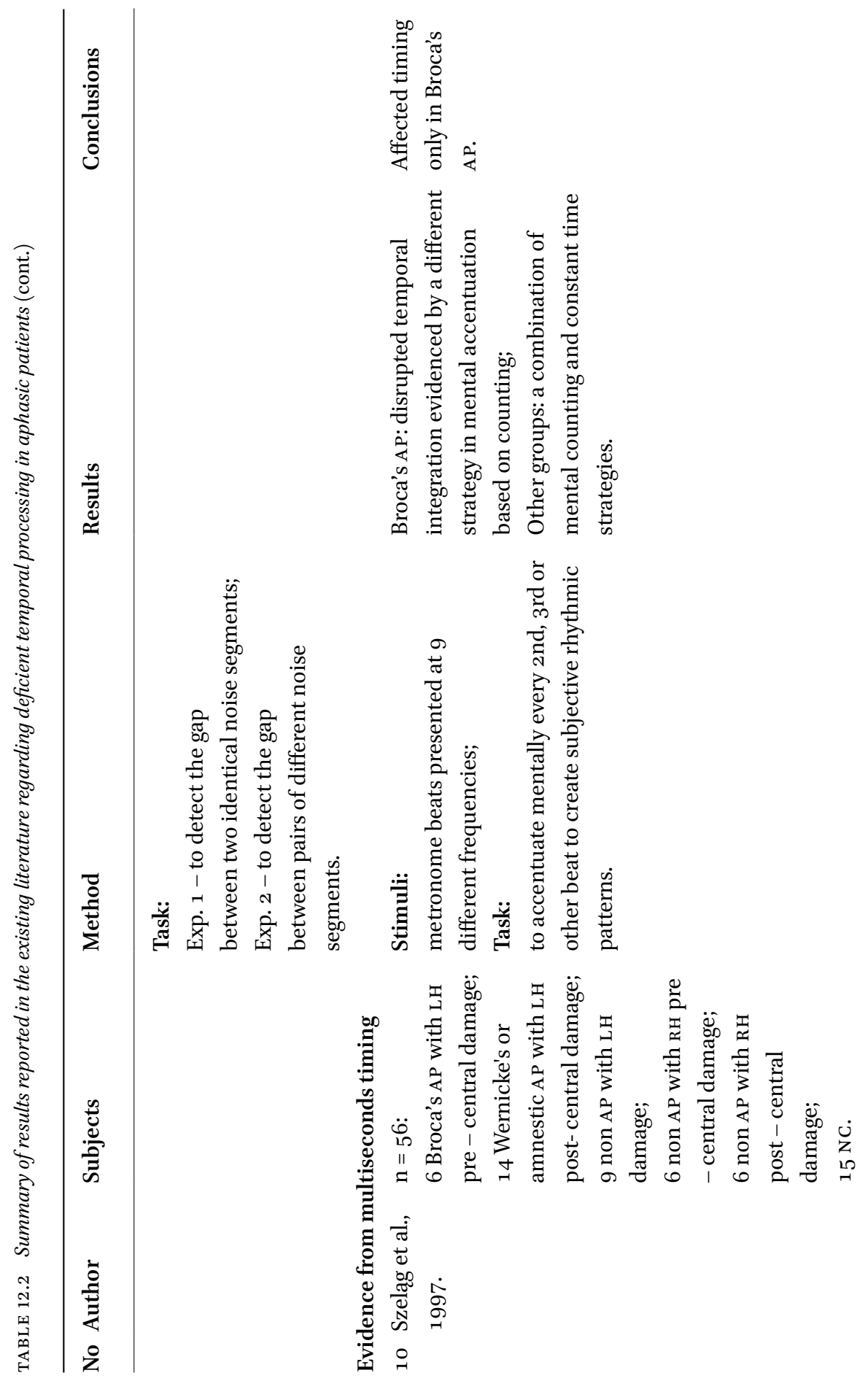



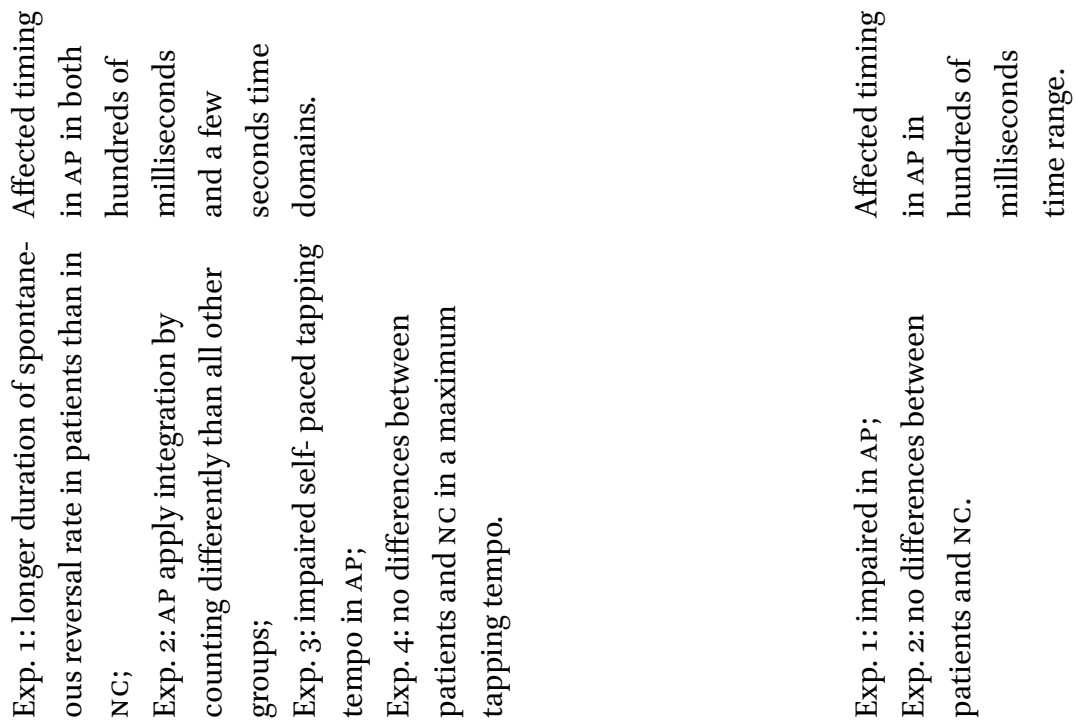

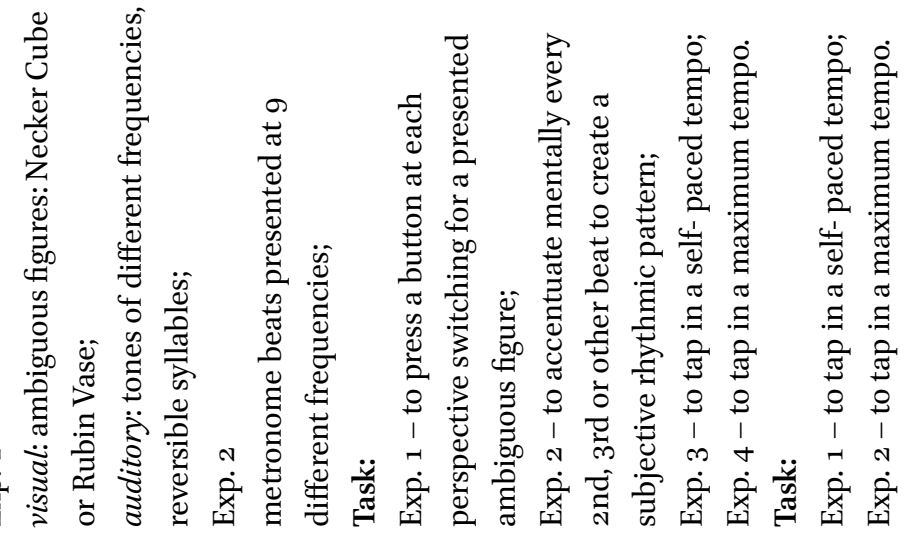
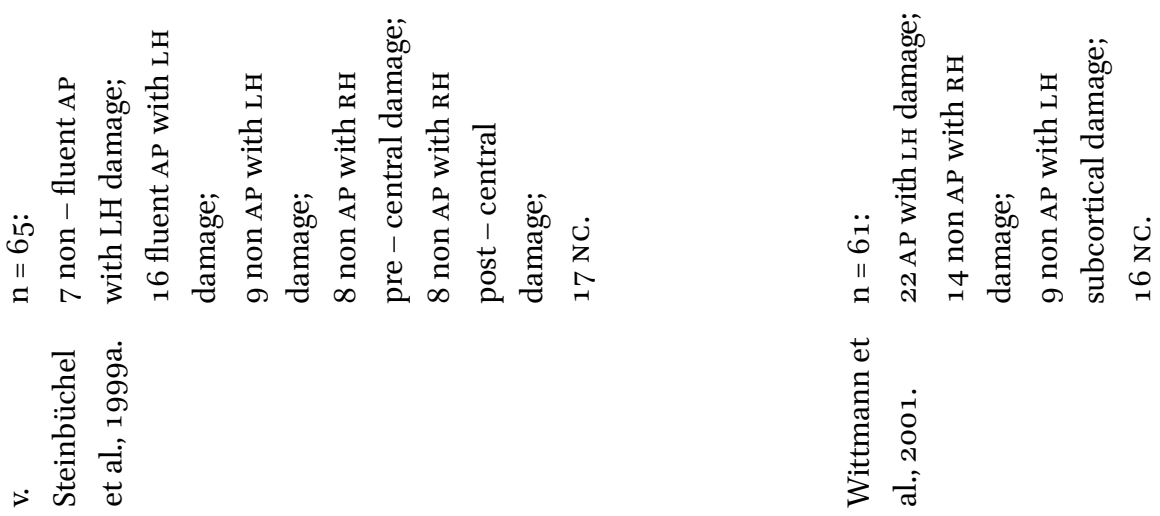


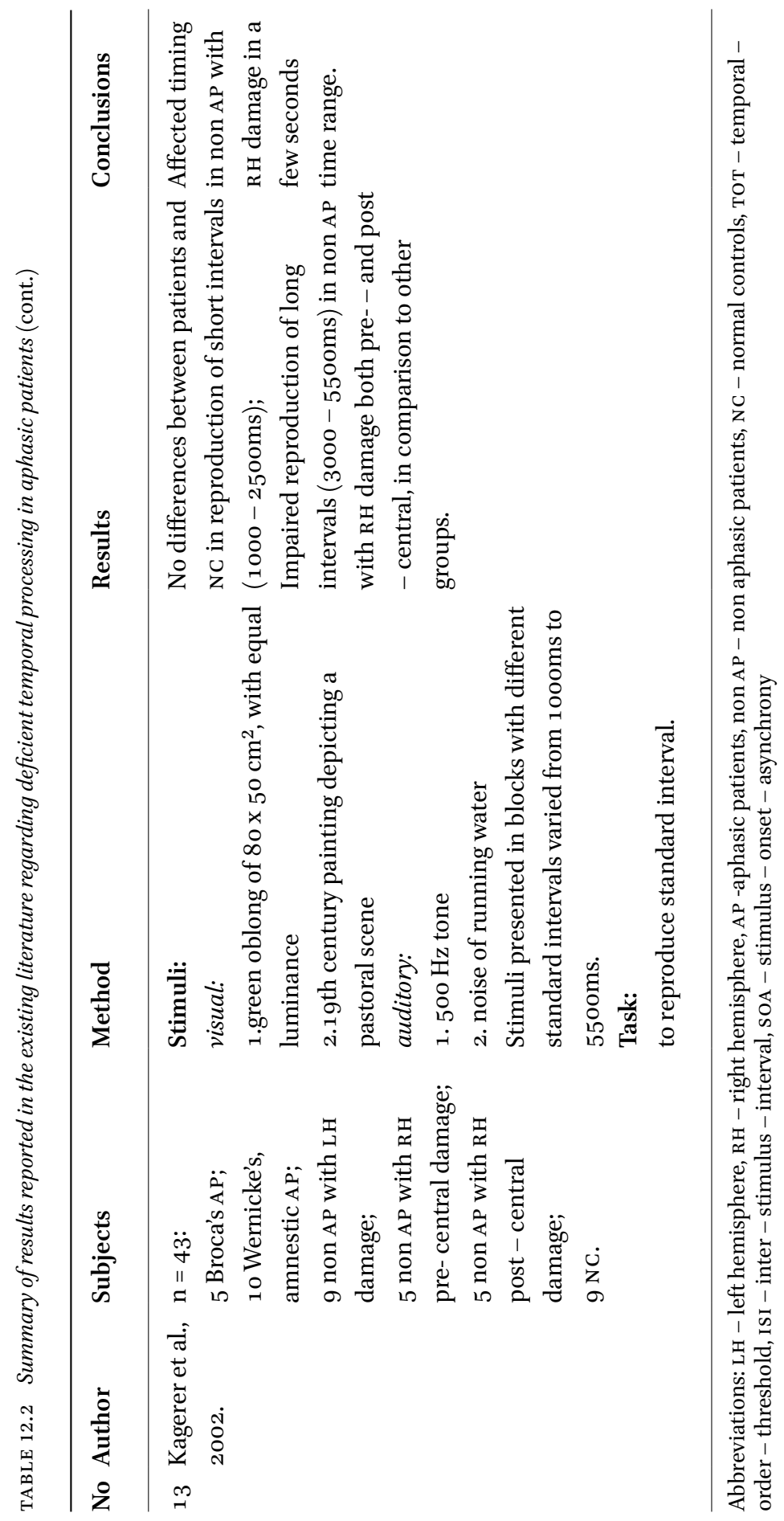


Despite the general features of neuroanatomical basis of language described above, a vast number of subsequent studies using various methods have confirmed the contribution of many additional areas of both hemispheres, as well as subcortical structures to verbal communication.

Taking into account the other language-sensitive regions, the traditional model of language representation assuming two well-defined parts supporting expression and reception needs to be modified. Although it seems very useful in the clinical practice and there is a number of evidence supporting such dissociation of language function, the current thinking on the traditional model of language is undergoing some fine-tuning, considering three groups of evidence: (1) neuroimaging data indicating the involvement of many other brain regions, beside the Broca's and Wernicke's areas, in language communication; (2) individual differences in representation of language production and reception far beyond the Broca's and Wernicke's area; (3) neuroanatomical representation of various language functions (see above) and phenomena, like bilingualism or sign language.

For instance, using neuroimaging it is possible to define the exact areas damaged by stroke or trauma and to relate these structural lesions to deficient language functions, which often do not cover the deficits expected by the traditional model. Moreover, data from individual patients indicated that lesions to the Broca's area not always led to Broca's aphasia. The deficits characteristic for this syndrome were also observed in case of brain damage to many other brain regions far beyond the region traditionally associated with the production of speech. Similar observations concern Wernicke's aphasia and disrupted auditory comprehension. It is interesting to note that brain imaging techniques (MRI, fMRI or PET) have revealed that anatomical damage does not necessarily cover the functional damage, which usually extends beyond the border of structural damage. At this point it should be mentioned that these neuroimaging techniques can map brain activity, but not necessarily linguistic function directly.

In summary, many brain regions control language communication. It would be a question for further studies what is their contribution to particular language functions for verbal communication, moreover, to what extent these areas are specific to language. To answer these questions, many psycholinguists seem to concentrate not only on the structures (localization) of particular language functions, but also on the neural mechanisms underlying language processing in real time, thus, on the temporal structure of linguistic processing. Focusing on temporal mechanisms underlying different language functions would allow us to understand temporal coordination, segmentation and integration involved in expression and comprehension. In this approach, studies 
on TIP in different time domains can indicate the activity of the dynamic network in which human language is rooted in norm and pathology. If the activity of the timing mechanisms operating at different ranges differs reliably in patients and controls, it may interfere with the associated mental activity.

Both everyday observations and experimental data indicate temporal constraints of human speech. Its temporal organization is multileveled. There is strong evidence supporting the thesis that our language communication is rooted in TIP, i.e., in temporal 'integration' and temporal 'chunking' or 'segmentation' on several time scales. One can distinguish the milliseconds (shorter time scale) and multiseconds (longer time scale) temporal constraints, which are controlled by a high- or low-frequency processing system, respectively (Pöppel, 2004; Szelag et al., 2004; Szelag, 2011). The former system concerns predominantly phonological encoding/decoding and syllabification, whereas the latter one concerns rather lexical selection and sentence production/reception.

It is important to note that language is not an isolated system, therefore, similar time scales can be distinguished in several key aspects of our behaviour, e.g., in motor control, as well as in many other mental functions which are strongly related to speech expression and/or reception (Pöppel 1997; Szelag et al., 2009). Therefore, we are of the opinion that timing is a critical factor for our verbal communication and provides an important insight into how the brain parses language in norm and pathology.

It is commonly known that normal production and understanding of language involves highly skilled coordination of several processes. Similar temporal levels or 'processing platforms' are incorporated in both speech expression (encoding) and reception (decoding). In phonological encoding, for instance, we produce the phonological forms, in particular, their segmental structure. Thus, central to phonological encoding are phonemes or syllables as the basic units for articulation produced by the speaker successively. On the other hand, in phonological decoding these phonological forms have to be perceived by the listener. Thus, these strings of successive phonemes and syllables perceived by the listener are also central to phonological decoding and speech reception.

The processing of temporal information in the speech signal has been investigated explicitly over the last few decades. The high-frequency processing system, i.e., a time domain of some tens of milliseconds, seems to be relevant to phonemic awareness (labelled also as phonemic hearing). It is defined as a 
unique human ability to analyse and synthesise speech sounds. These processes are fundamental with respect to auditory comprehension. In the time platform of about 20-40ms, information about the place of articulation in stopconsonants ( $\mathrm{p}, \mathrm{b}, \mathrm{t}, \mathrm{d}$ etc.) is contained. Formant transitions, characterized as short sound waveforms that change frequency across a time interval of ca. $40 \mathrm{~ms}$ vary, according to the place of articulation. Spectrographic analyses of our verbal utterances clearly indicated that rapid formant transitions in stopconsonants ( $\mathrm{p}, \mathrm{b}, \mathrm{t}, \mathrm{d}$ etc.) are limited in time up to 4oms. Because of the specific structure of our articulators these stop-consonants cannot be prolonged in the fluent speech because a forthcoming vowel sounds immediately.

On the other hand, fricatives ( $\mathrm{w}, \mathrm{f}, \mathrm{s}, \mathrm{z}$ etc.) or vowels (a, o, u etc.) are characterized by a structure less limited in time and can sound in the fluent speech much longer, even up to 20oms. Despite important individual differences in the speech expression rate, particular speech sounds come on average as fast as 10 to 15 phonemes per second. The temporal structure of the verbal output is usually automatic (controlled by procedural memory) and often happens without any attentional or motor control of 'how' we say things. In contrast, 'what' we say is fully consciously controlled.

Another example of temporal constraints with respect to phonemic awareness on the millisecond time scale comes from the Voice-Onset-Time (VоT) paradigm. In the perception of voicing contrasts of stop-consonants in syllables like DO $v s$. TO, a difference in duration of about 4oms between the burst of the air and the onset of laryngeal pulsing in articulation of the initial consonant, is defined as the vот. It distinguishes voiced $(/ \mathrm{b} /, / \mathrm{d} /, / \mathrm{g} /$ etc.) from voiceless stop-consonants (e.g., /p/, /t/, / k/ etc.).

Such evidence supports the thesis that the high-frequency processing system is related to phoneme processing and phonemic hearing, thus, the crucial processes with respect to auditory comprehension.

Furthermore, there exists the low-frequency processing system, corresponding to the time range of a few seconds, which is related to the duration of sentences in fluent speech. For example, our average syllabic rate is ca. 3-4 per seconds, whereas words are usually articulated at a speed of approx. 2 per second. At this rate we are able to retrieve lexical items from a mental lexicon that on average contains probably tens of thousands items. Although there are some so-called 'fast speakers' who are able to produce even up to 7 words per second, it is usually difficult to follow such fast speech tempo, which does not fit the typical tempo.

Another source of evidence comes from analysis of the temporal structure of fluent speech in many languages, like Polish, Russian, German, English or Chinese (Pöppel, 1997). Several data have indicated that in many languages the duration of phrases in fluent speech is limited in time up to a few seconds. 
Thus, spontaneous flow of speech is temporally segmented or 'chunked' in a few seconds time domain. This phenomenon is called 'semantic parsing'. Usually, particular phrases are followed by hesitation pauses that are necessary both for the listener to process the information provided by the speaker, as well as for the speaker to prepare the next phrase.

At this point it seems interesting to note that similar temporal segmentation as observed in oral language has been confirmed in sign language. In such way of communication, consecutive signs (i.e., motor acts) are grouped within segments of a few seconds duration, followed by pauses when signs are not provided. The duration of particular signed segments corresponds to those observed in oral language, described above, with a little shorter durations of ca. $2 \mathrm{~s}$ in sign language. The similar temporal chunking of speech expression, independently of the kind of articulation (using either the oral output or hand movements) suggests the existence of a common neural mechanism controlling human language communication, independently of the output from the system.

To summarize, both the millisecond and multisecond time ranges are crucial for our language communication. Hence, authors are of the opinion that the left hemispheric specialization for language derives from specialization for timing. Furthermore, language deficits of various etiologies in children and adults have been associated with timing disorders on both mentioned time scales. Language processing depends, thus, on temporal constraints of the brain on several levels, which may be affected after the brain injury.

In the traditional view different language disorders (e.g., aphasia, specific language impairment) or reading and writing disorders (dyslexia) have been viewed as distinct clinical syndromes. Recently, this viewpoint has been reformulated because of a close link between these syndromes. Neuropsychological profiles taking into account the coexistence of specific temporal processing disorders and phonological impairments show some similarities between particular syndromes. In general, they are characterized by deficient TIP in both, the millisecond and multisecond ranges. Because of the central relevance of TIP to our understanding of the neural basis of cognitive systems, one may define a typical TIP and abnormalities in such processing in human populations suffering from impaired cognitive function (Szelag and Skolimowska, 2012).

\section{6}

\section{Deficits in Processing Temporal Information in Aphasic Patients}

Studies of patients suffering from aphasia following left hemispheric brain damage provide an important contribution to our understanding of TIP. 
The first evidence of this type was provided by Efron (1963) and later by Swisher and Hirsh (1972). They observed that aphasic patients displayed parallel deficits in both speech comprehension and rapidly changing temporal information.

These observations were confirmed in several further reports, including our own studies. Table 12.2 summarized the results of studies conducted using different patient populations and experimental techniques. Looking at Table 12.2, we may conclude that TIP within both the millisecond (points 1-9) and multisecond (points 10-13) time ranges is usually disturbed in patients with aphasia following left hemispheric brain damage.

There exist also some experimental evidence on the high frequency processing system in normal healthy volunteers. These studies are often focussed on temporal order of incoming events and measurement of temporal-order threshold. It reflects an ability to perceive the relation before-after for stimuli presented in rapid succession in order to be brought into a sequence by approximately the same value of some tens of milliseconds for visual, auditory, and tactile modality (Hirsh and Sherrick 1961; Kanabus et al., 2002; Zampini et al., 2003, 2005). The correspondence of this value across different senses suggests a common central mechanism for vision, hearing or touch and it may be concluded that the neural process underlying sequential ability is activated to identify primordial events, independently of the sensory modality (Pöppel, 2009). Such ability allows us to reduce complexity of incoming events, which stimulate incessantly our senses, and to create our conscious percepts.

Strong experimental evidence provided in Table 12.2 (points 1-9) may suggest a disruption of this high-frequency process following left hemispheric brain damage, resulting in disordered language communication, as a consequence of primordial event disruption. Moreover, the data on temporal constraints of human speech on the milliseconds level discussed in the previous section have indicated the importance of this time platform with respect to speech reception. Hence, disordered processing of information on this level following brain injury may underlie language problems, especially, problems in phonemic hearing and auditory comprehension. These problems often constitute a core symptom after posterior lesions to the left hemisphere, resulting in receptive aphasia (see above for aphasia classification).

On the other hand, Table 12.2 presents also data indicating deficient timing on low frequency processing, i.e., in the domain of hundreds of milliseconds or a few seconds (points 9-13). Considering temporal constraints of speech (see Section 5), the former time window is related to the level of syllables, whereas, the latter one may be important with respect to the fluent verbal output and expression of phrases and sentences. It requires the ordering of successive 
syllables and words into logic strings, which build fluent utterances and provide a structure for language communication.

According to the hierarchical model of time perception proposed by Ernst Pöppel (1997, 2004; Szelag et al., 2004), the existence of temporal integration on a few seconds scale creates a high-frequency level of complexity reduction. In fact, in fluent verbal communication we usually do not concentrate so much on particular phonemes, syllables or words, but on logic utterances of what we say or what we hear. Thus, the primordial events identified on a level of higher temporal resolution (some tens of milliseconds) are next sequentially linked together into a perceptual 'gestalt', representing an operating range of a few seconds. This process is also labelled in the literature as 'temporal binding' or 'temporal integration' and it is expected to be important for our conscious experience, of which verbal communication may be one example.

Although reports on TIP on the supra-second level are fewer, Table 12.2 shows that using different experimental paradigms, like subjective accentuation of metronome beats (points 10-11), spontaneous reversal rate of ambiguous figures (point 11), finger tapping in a self-pace and maximum tempi (point 12) or reproduction of temporal intervals (point 13), a disruption of temporal binding can be observed, which has been also evidenced following injury to the brain, predominantly to the anterior parts of left hemisphere, resulting in motor aphasia. The patients suffering from this syndrome had deficient binding operations that probably underlie problems with the fluent verbal output (i.e., effortful, nonfluent speech, compare above), but also some comprehension deficits, especially for longer utterances, which need to integrate and hold the incoming information for a few seconds.

To sum up, acquired language disorders following brain damage are strongly related to deficient timing on several levels, which create a neural timeframe for verbal communication. Thus, the superiority of the left hemisphere for the processing of verbal information may reflect more primary specialization for TIP.

\section{7 \\ Relationships between Disrupted Timing and Specific Aphasia Syndromes}

It seems also interesting to note, that in our studies on disrupted timing in aphasia, an important dissociation between specific language deficits (in phonemic hearing $v$ s. effortful nonfluent verbal output) and temporal deficits (at the sub- or supra-second level) were observed (v. Steinbüchel et al., 1999b; Szelag et al., 1997; Wittmann et al., 2001). Specific left hemispheric lesions selectively damaged temporal mechanisms operating either within the millisecond or multi-second time scales (Table 12.2, points 4-6, 10-12). The former 
range was assessed with auditory temporal-order-threshold (Figure $\mathbf{1 2 . 2}$ whereas, the latter one with subjective accentuation paradigm, which corresponded to the upper limit of the temporal integration mechanism, operating in a time window of a few seconds (Figure 12.3). In patients with left hemispheric post-central lesions, suffering from Wernicke's aphasia (characterized by deficits in phonemic hearing and poor comprehension), deficient temporal-order-thresholds were reported (Figure 12.2). Those patients needed a significantly longer gap between successive events to correctly report their before-after relation. These timing deficits corresponded to their language

\section{Auditory Order Threshold}

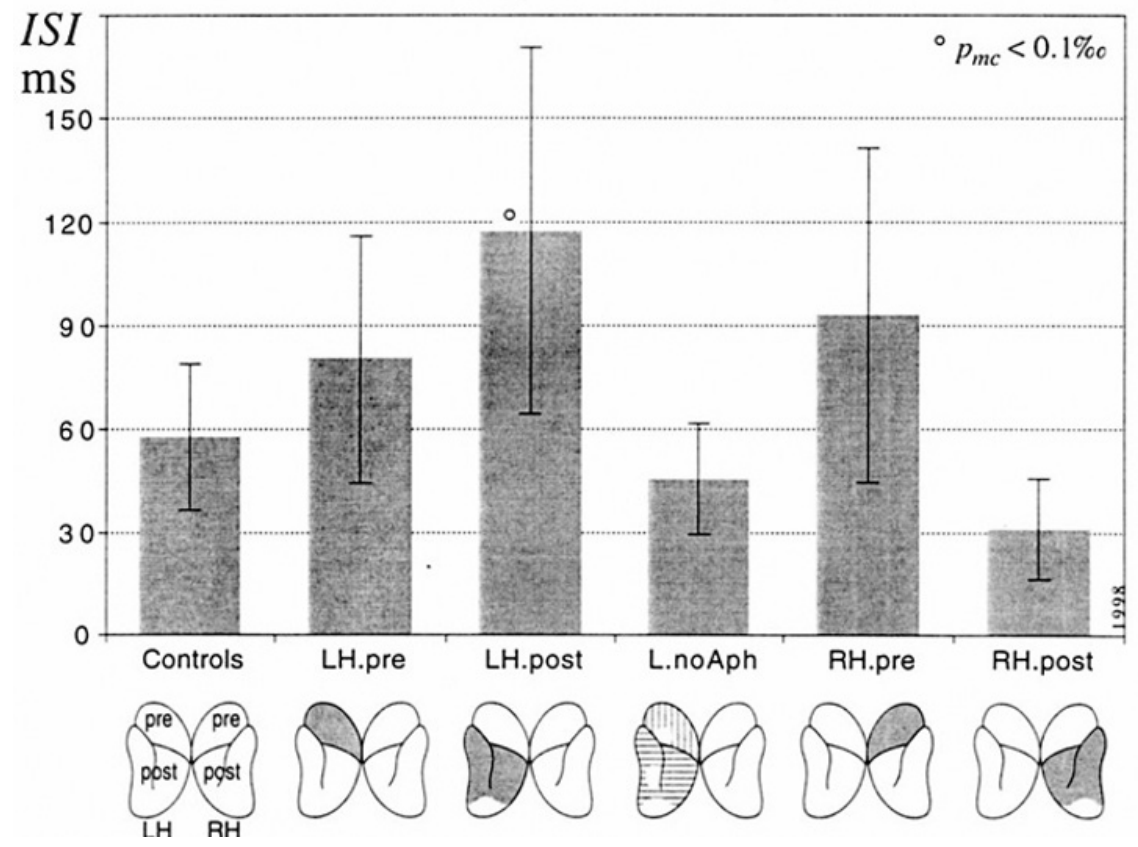

FIGURE 12.2 Auditory order thresholds (mean and standard deviations) for the five patient groups with focal brain injuries and for an orthopaedic control group are shown: LH. pre-anterior left hemisphere (pre-central) with non-fluent aphasia; LH. post-posterior left hemisphere (post-central) with fluent aphasia $\left({ }^{*} p<1 \%\right.$ for group differences as compared with controls and L. noAph; statistical calculation with Scheffe post-hoc test); L. noAph-left-sided subcortical lesions without aphasia; $R H$. pre-anterior right hemisphere (pre-central); $R H$. post-posterior right hemisphere (post-central). Reprinted from Neuroscience Letters, 264, v. Steinbüchel N., Wittmann M., Strasburger H., Szelag E. "Auditory temporal-order judgment is impaired in patients with posterior regions of the left hemisphere" $168-71$.

COPYRIGHT (1999) WITH PERMISSION FROM ELSEVIER 


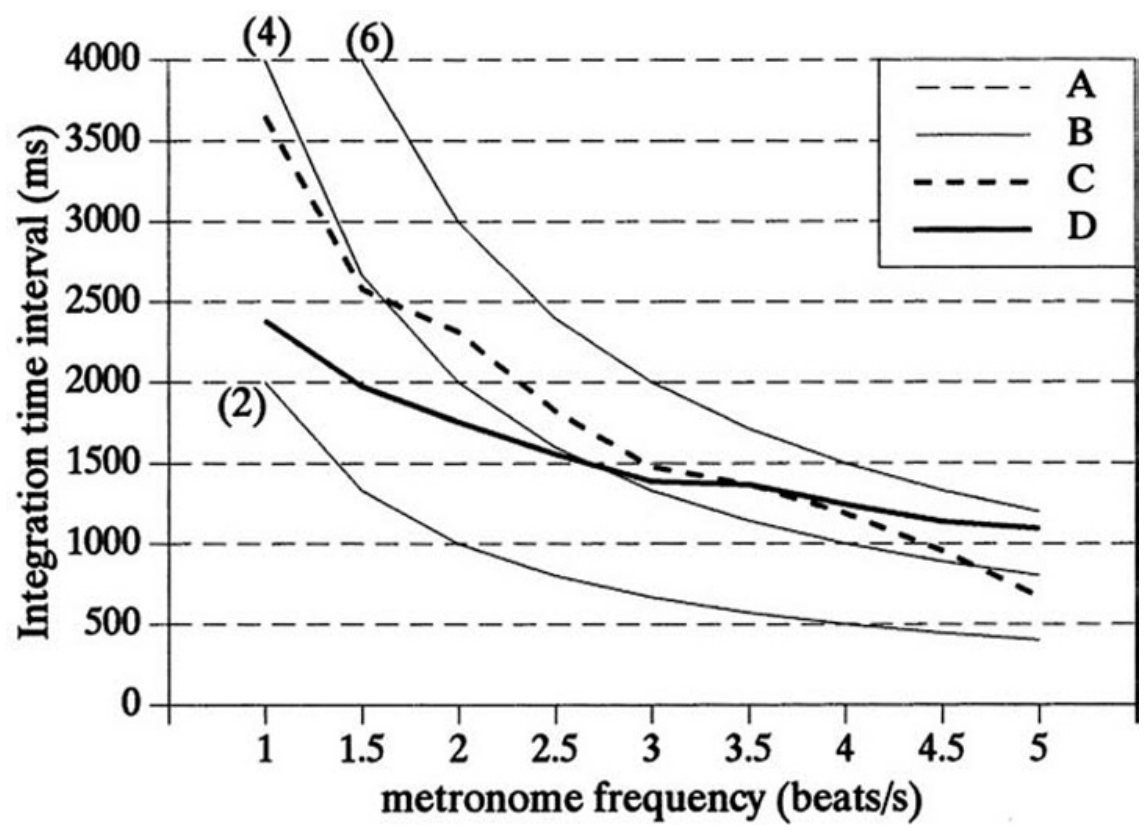

FIGURE 12.3 The measured integration interval length (MIIL) plotted against the metronome frequency using three different strategies in Broca's aphasia and other patients. Integration $(A)$ by time; $(B)$ by number; $(C)$ in Broca's aphasic patients; $(D)$ in all the remaining patients. Standard deviation values (in $m s$ ) for the consecutive frequencies in the Broca's aphasic patients: 1964, 911, 887, 591, 439, 363, 408, 491, 406; in the other subjects: 1191, 698, 535, 370, 338, 370, 363, 394, 401. Reprinted from Neuroscience Letters, 235, Szelag E.v. Steinbüchel N., Pöppel E. "Temporal processing disorders in patients with Broca's aphasia" $33-36$.

COPYRIGHT (1997) WITH PERMISSION FROM ELSEVIER

deficits, because limited duration of consonants and vowels in incoming words could not be properly decoded because of disrupted timeframe.

In contrast, Broca's aphasic patients were unaffected on this high processing level (Figure 12.2). They were characterized by relatively preserved comprehension but nonfluent speech and displayed deficits within the supra-second processing range (Figure 12.3).

Using subjective accentuation paradigm of monotonous metronome beats, normal healthy volunteers can integrate the information up to a maximum of ca. 3 seconds. In this task they applied a combination of integration strategies based on perceptual grouping of separate beats in a constant time (related to the upper limit of temporal integration, Figure 12.3 dashed parallel lines A reflecting integration within e.g., 1000, 2000 or $4000 \mathrm{~s}$ time windows) and automatic counting up to 2, 4 or $6 \mathrm{~s}$ (Figure 12.3 solid lines B labelled by 
numbers 2,4 or 6 ). In contrast, in Broca's aphasic patients the constant time strategy was disrupted, but the accentuation was based predominantly on automatic mental counting (Figure 12.3 bold dashed line C). Moreover, the upper limit of integration in Broca's aphasic patients exceeded the typical frames characteristic for normal healthy volunteers. This suggests deficient temporal integration in motor aphasic patients in a time window of a few seconds, but relatively preserved automatic mental counting. The data obtained implied that some highly structured syntactic abilities related to temporal integration are located in the anterior language area (Broca's area).

The evidence provided by Szelag et al. (1997) and v. Steinbüchel et al. (1999); Table 12.2 point 10-11; Figure $\mathbf{1 2 . 2}$ and 12.3) showed that a disruption in temporal binding is a core symptom in case of nonfluent speech and agrammatism, dominating in motor aphasia. In fact verbal expression requires information to be integrated and held for a few seconds. The disruption of temporal integration at the level of seconds may cause symptoms typical for motor aphasia.

Furthermore, aphasic patients demonstrated impaired self-paced (personal) tempo of finger tapping, indicating the dominant control of left hemisphere in voluntarily timed actions in the special time scale of hundreds of milliseconds involved in motor behaviour (Wittmann et al., 2001; Figure 12.4). These timing deficits may be related to disrupted TIP linked to the syllable level, independently of the type of aphasia.

To conclude, we argue that TIP in aphasics may be selectively affected at either sub- or supra-second time range, depending on the localisation of lesion and observed disfluency pattern. We also postulate that a disruption of timing mechanisms leads to phonological and/or syntactical disorders, commonly observed in aphasic patients.

These results may have some practical applications with respect to new neurorehabilitation methods. As TIP processing is impaired in aphasic patients, in our recent studies we verified the effectiveness of a new method of aphasia therapy which is concentrated not on the linguistic level, but on the pre-semantic one and concerns the training of neuronal mechanisms underlying both TIP and language (Szelag et al., 2014). These methods address both the receptive and expressive language functions, in particular, auditory speech comprehension, phonemic hearing and nonfluent verbal output.

Our recent studies are addressed greater understanding of the relationship between timing and cognition (Szelag et al., 2011). Using Fast For Word Language $^{\circ}$ (FFW) program, we showed for the first time significant benefits of such temporal training on broad aspects of cognitive function in the elderly beyond 65 years of life (Szelag and Skolimowska, 2012). Following such training, we indicated stable positive effects not only in TIP, but also in divided and 
Maximum-tapping tempo

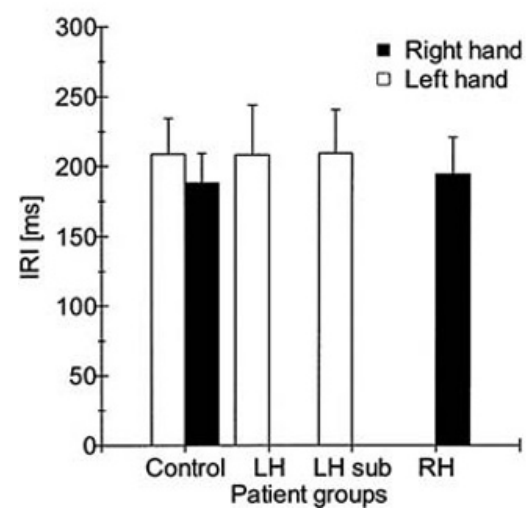

Personal-tapping tempo

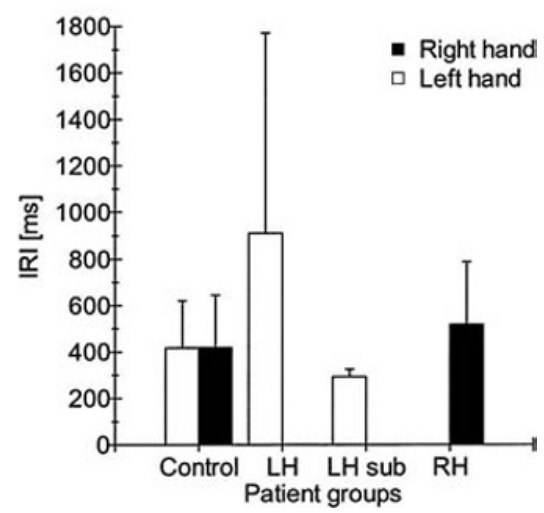

FIGURE 12.4 Maximum-tapping tempo and personal-tapping tempo as mean inter-response interval (IRI) over the orthopaedic control group and the brain-injured patient groups with left-hemispheric cortical lesions $(L H)$, with left-hemispheric lesions in predominantly subcortical regions ( $L H$ sub) and with right-hemispheric cortical lesions $(\mathrm{RH})$. Data for the brain-injured patients are only presented for the hand ipsilateral to the lesion site. Reprinted from Cognitive Brain Research, 10, Wittmann M., v. Steinbüchel N., Szelag E. "Hemispheric specialisation for selfpaced motor sequences" $341-44$. COPYRIGHT (2001) WITH PERMISSION FROM ELSEVIER

vigilance of attention, matching complex patterns, and working memory span. These results show a new impact of temporal training not only on deficient language function, but also on age-related cognitive decline in the senior population.

On the basis of these results we offer a promising direction for neurorehabilitation and have recently initiated an innovative rehabilitation computer program that addresses improvement of a broad range of cognitive functions in children and adults.

Aphasia is a complex language disorder and refers to deficits apparent in speech, writing, and reading caused by an injury to brain areas, specialized for these functions. It is commonly known that the expression and reception of language have defined temporal dynamics and involve highly skilled coordination of several processes. Language processing depends, thus, on the 
temporal constraints of information processing which may be affected after injury to the brain.

In this chapter we summarize the existing literature studies on TIP as a key to understand language disturbances in aphasic syndromes. The literature data are illustrated with examples from our studies, indicating parallel language and timing deficits following damage to specific brain regions. We showed dissociation between receptive and expressive language deficits associated with a disruption of specific timing mechanisms controlling information processing on milliseconds or multiseconds time domains.

To conclude, in aphasic patients TIP may be selectively affected either within the sub- or supra-second range, depending on the lesion location and observed disfluency pattern. A disruption of timing mechanisms leads to phonological disorders and/or effortful nonfluent speech. Finally, we have indicated that the specific nonverbal training in temporal processing can ameliorate auditory comprehension in aphasic patients. These studies provide an important contribution to our understanding of TIP as a neural basis of language, as well as other cognitive function.

\section{Acknowledgments}

Supported by grants 507/1/N-DFG/2009/o and INNOTECH/IN1/30/159041/ NCBR/12. We wish to thank Argiro Vatakis and Melissa Allman for the helpful reading of this chapter.

\section{References}

Blanken, Gerhard et al. 1993. Linguistic Disorders and Pathologies, Berlin - New York (Walter de Gruyter).

Efron, Robert. 1963. "Temporal Perception, Aphasia and Déjà vu" Brain 86: 403-24.

Fink, Martina, Jan Churan, and Marc Wittmann. 2006. "Temporal Processing and Context Dependency of Phoneme Discrimination in Patients with Aphasia" Brain and Language 98: 1-11.

Hirsh, Ira J., and Carl E. Sherrick. 1961. "Perceived Order in Different Sense Modalities" Journal of Experimental Psychology 62: 423-32.

Kagerer, Florian A. et al. 2002. "Cortical Involvement in Temporal Reproduction: Evidence for Differential Roles of the Hemispheres” Neuropsychologia 40(3): $357-66$. 
Kanabus, Magdalena et al. 2002. "Temporal Order Judgement for Auditory and Visual Stimuli" Acta Neurobiologiae Experimentalis 62: 263-70.

Osterhout, Lee. 2000. "On space, time, and language: For the next century, timing is (almost) everything" Brain and Language 71: 175-7.

Penfield, Wilder, and Lamar Roberts. 1959. Speech and Brain Mechanisms, New York: Princeton University Press.

Pöppel, Ernst. 1997. "A Hierarchical Model of Temporal Perception" Trends in Cognitive Sciences 1: 56-61.

. 2004. "Lost in time: A historical frame, elementary processing units and the 3-second window" Acta Neurobiologiae Experimentalis 64: 295-302.

- 2009. "Pre-Semantically Defined Temporal Windows for Cognitive Processing" Philosophical transactions of the Royal Society, Series B, Biological Sciences 364: 1887-96.

Price, Cathy. 2004. "An Overview of Speech Comprehension and Production" In Human brain function edited by Richard Frackowiak, Karl J Friston, Christopher D Frith. Raymond J Dolan, Cathy Price, Semir Zeki, John T. Ashburner and William D Penny, 517-32. Oxford: Elsevier.

Purves, Dale et al. 2008. Principles of Cognitive Neuroscience Sunderland-Massachusetts USA: Sinauer Assoc., Inc. Publishers.

Scott, Sophie K. 2012. "The neurobiology of speech perception and production - can functional imaging tell us anything we did not already know?" Journal of Communication Disorders 45: 419-25.

Sidiropoulos, Kyriakos et al. 2010. "Temporal Processing Capabilities in Repetition Conduction Aphasia" Brain and Cognition 73(3): 194-202.

Springer, Sally P., and Georg Deutsch. 1989. Left Brain, Right Brain New York: H. Freeman and Co.

Stefanatos, Gerry A. et al. 2007. "Fine Grain Temporal Analysis in Aphasia: Evidence from Auditory Gap Detection" Neuropsychologia 45(5): 1127-33.

Swisher, Linda, and Ira J. Hirsh. 1972. "Brain Damage and the Ordering of Two Temporally Successive Stimuli" Neuropsychologia 10: 137-52.

Szaflarski, Jerzy P. et al. 2011. "Poststroke Aphasia Recovery Assessed with Functional Magnetic Resonance Imaging and a Picture Identification Task" Journal of Stroke and Cerebrovascular Disease 20(4): 336-45.

Szelag, Elzbieta et al. 2004. "Individual Differences in Temporal Information Processing in Humans" Acta Neurobiologiae Experimentalis 64: 349-66.

Szelag, Elzbieta et al. 2008. "Cortical Representation of Time and Timing Processes" In Neuronal correlates of thinking, edited by Eduardo Kraft, Balazs Guylas and Ernst Pöppel, 185-99. Berlin, Springer-Verlag.

Szelag, Elzbieta et al. 2011. "Hearing Loss and Auditory Processing Disorders: Clinical and Experimental Perspectives" In Culture and Neural Frames of Cognition and Communication edited by Shihui Han and Ernst Pöppel, 153-68. Berlin: Springer. 
Szelag, Elzbieta et al. 2009. "Neuropsychological Rehabilitation from Brain-Behavior Perspective" In New Ideas in Studying and Supporting Development of Exceptional People edited by Ewa Pisula and Piotr Tomaszewski, 49-63.Warszawa:Wydawnictwo Uniwersytetu Warszawskiego.

Szelag, Elzbieta, Nicole v Steinbüchel, and Ernst Pöppel. 1997. "Temporal Processing Disorders in Patients with Broca's Aphasia" Neuroscience Letters 235:33-6.

Szelag, Elzbieta, and Justyna Skolimowska. 2012. "Cognitive Function in Elderly Can be Ameliorated by Training in Temporal Information Processing" Restorative Neurology and Neuroscience 30: 419-34.

Szelag, Elzbieta et al. 2014 "Training in Temporal Information Processing Ameliorates Auditory Comprehension in Aphasic Patients: A Randomized Controlled Pilot Study" Journal of the Neurological Sciences 338: 77-86.

Tallal, Paula, and Freda Newcombe. 1978. "Impairment of Auditory Perception and Language Comprehension in Dysphasia" Brain and Language 5: 13-24.

Turken, And U., and Nina F. Dronkers. 2011. "The neural architecture of the language comprehension network: converging evidence from lesion and connectivity analysis" Frontiers in Systems Neuroscience 5(1): 1-20.

von Steinbüchel Nicole, Marc Wittmann, and Elzbieta Szelag. 1999a. "Temporal Constraints of Perceiving, Generating and Integrating Information: Clinical Evidence" Restorative Neurology and Neuroscience 14: 167-82.

von Steinbüchel Nicole, . 1999b. "Auditory Temporal-Order Judgement is Impaired in Patients with Cortical Lesions in Posterior Regions of the Left Hemisphere" Neuroscience Letters 264: 168-71.

Wittmann Marc et al. 2004. "Effects of Brain-Lesion Size and Location on Temporal Order Judgement" Neuroreport 15(15): 2401-5.

Wittmann Marc Nicole v Steinbüchel, and Elzbieta Szelag. 2001. "Hemispheric Specialisation for Self-Paced Motor Sequences" Cognitive Brain Research 10: 341-4.

Zaidel, Eran. 2001. "Hemispheric Specialization for Language in the Split Brain" In Handbook of neuropsychology: Language and aphasia, edited by François Boller and Jordan Grafman, 393-418. Amsterdam: Elsevier.

Zaidel, Eran et al. 2003. "The Callosal Syndromes” In Clinical Neuropsychology edited by Kenneth M Heilman and Edward Valenstein, 347-403 (4th edition). New York: Oxford University Press, Inc..

Zampini, Massimiliano, David I. Shore, and Charles Spence. 2003. "Audiovisual Temporal Order Judgments" Experimental Brain Research 152(2): 198-210.

Zampini, Massimiliano et al. 2005. "Audiotactile Temporal Order Judgments" Acta Psychologica 118(3): 277-91. 